\title{
Detailed non-destructive evaluation of UHMWPE composites in the terahertz range
}

\author{
N. Palka - D. Miedzinska
}

Received: 17 May 2013 / Accepted: 9 November 2013 / Published online: 19 November 2013

(C) The Author(s) 2013. This article is published with open access at Springerlink.com

\begin{abstract}
We report on the terahertz analysis of an internal structure of an ultra-high molecular weight polyethylene (UHMWPE) composite material, which is based on the HB10-tape from Dyneema ${ }^{\circledR}$. This type of composite is very hard and resistant and therefore it is often used to manufacture personal armors such as bulletproof vests and helmets. The multilayer structure of the UHMWPE composite was investigated by means of a raster scanning time domain spectroscopy technique in a reflection configuration. The mechanism of the formation of many shifted in time $\mathrm{THz}$ pulses (reflected from the internal layers of the sample) originates from the periodic modulation of the refractive index along the propagation of the radiation. This modulation is connected with alternate layers of fibers, each having different direction (perpendicular to each other). As a result we obtained the detailed three dimensional profile of the 3.3-mm thick sample with all 74 layers clearly visible. Thicknesses of all layers, having around $45 \mu \mathrm{m}$ each, were determined. Moreover, it is also possible to identify internal defects i.e. delaminations in the internal structure of this composite material.
\end{abstract}

Keywords Terahertz imaging - Time domain spectroscopy - Nondestructive testing . UHMWPE composites

\section{Introduction}

Composite materials are widely used in many areas of technology, including civil engineering, aerospace and astronautics (e.g. aircraft parts, missiles, satellites), the road and rail transport industry, the production of machinery, equipment and sporting goods. Especially they are important in the military applications as protective materials for both, soldiers and vehicles.

\footnotetext{
N. Palka (凶)

Institute of Optoelectronics, Military University of Technology, 2 Kaliski Str., Warsaw, Poland e-mail:npalka@wat.edu.pl

D. Miedzinska

Faculty of Mechanical Engineering, Military University of Technology, 2 Kaliski Str., Warsaw, Poland
} 
Despite many advantages over conventional materials, composites may have defects such as voids, delaminations, matrix and fibers cracks, punctures, mechanical damages or heat damages (Stoik et al. 2010). All these imperfections are associated with the production processes and degradation, which result from their maintenance in harsh and demanding environments. Defects in the structure of the composite can cause severe weakening of its properties. It leads to routine inspections of many elements, e.g. aircraft parts, using a number of complex techniques such as: thermographic (Levar and Hamilton 2003), ultrasonic (Gendron et al. 1995), acoustics and shearography (Dragan and Swiderski 2010), X-ray (Bathias and Cagnasso 1992) and microwave (Hughes et al. 2002). With regard to vast applications of composites, studies devoted to the development of new and better materials are being conducted and their properties must also be tested.

The rapid development of composite materials during the last decades has increased the demand for new non-destructive evaluation (NDE) solutions. Terahertz $(0.1-3.0 \mathrm{THz})$ imaging is novel visualization technique which can serve in many application areas, like detecting explosives and related compounds (Chan et al. 2007), identifying various defects (Lopato et al. 2009), tomography and materials identification and characterization (Mittleman et al. 1999) or detecting contaminations (Wietzke et al. 2007). Electromagnetic waves in the range of $0.1-3.0 \mathrm{THz}$ has an unique ability to penetrate many materials, which are opaque in the range of visible and infrared light, thus enabling visualization of their internal structure. Moreover, terahertz radiation offers non-destructive, contactless and nonionizing method of evaluation of some materials and could compete with the above mentioned techniques using other ranges of the electromagnetic radiation.

The THz time domain spectroscopy (TDS) is the most commonly used method from all $\mathrm{THz}$ techniques for non-destructive evaluation of composite materials (Chan et al. 2007; Lopato et al. 2009; Mittleman et al. 1999; Wietzke et al. 2007). Although other techniques based on a FMCW radar (Quast et al. 2010) as well as CW imaging by means of quantum cascade lasers (Destic et al. 2010), a gas laser (Redo-Sanchez et al. 2006) or with a Gunn diode (Karpowicz et al. 2005) were also studied.

Depending on the position of the transmitter and the receiver in relation to the sample we can distinguish two techniques - one using the configuration in transmission and the other in reflection. In general by using transmission methods the cumulative image of the sample is obtained and it is worth noticing that such image contains total information about the sample. The areas with different attenuation are observed and on the basis of their distribution the heterogeneities and defects of the internal structure can be detected. Moreover, the methods based on the TDS and the FMCW radar can work in the reflection mode. Due to this fact these systems can collect significantly more information about the structure of the multilayer sample, about the distribution and thickness of particular layers and about the type and position of various defects.

In the reflection TDS method the very short electromagnetic pulse propagates through the sample. Some parts of the pulse are reflected on the interfaces between the media having different refractive indices. In case of multilayer samples (like composite materials) the sequence of shifted in time pulses reflected from particular interfaces is registered. It enables the precise analysis of the internal structure (the time-of-flight imaging). This method can be applied to the examination of thin samples (in the range of $1 \mathrm{~cm}$ ), but with high axial resolution of about $30 \mu \mathrm{m}$ (minimum layer separation distance) or even smaller (Takayanagi et al. 2009). This resolution is proportional to the temporal width of the incoming pulse and inversely proportional to the group refractive index of the layer (Yasui et al. 2005). The other reflection method is using the modulation of the frequency of the continuous wave (FMWC) on the basis of which positions of the interfaces are determined. The former method can 
be applied to the examination of thicker samples (in the range of $5-15 \mathrm{~cm}$ ), but its axial resolution is only about $1-2 \mathrm{~mm}$ (Quast et al. 2010).

Taking into account the attenuation of different media only some composite materials can be examined in the $\mathrm{THz}$ range. Carbon fiber composites reflect very well the $\mathrm{THz}$ radiation and almost nothing penetrates to their internal structure (Redo-Sanchez et al. 2006). However, glass fiber reinforced composites, for which numerous examinations are carried out due to their universality of applications (Stoik et al. 2010; Rutz et al. 2006) have better parameters for the examination in the $\mathrm{THz}$ range, despite the problems with transmission and scattering of the radiation.

An ultra-high molecular weight polyethylene (UHMWPE) composites are less common and that is why we can point only one article cursory describing the topic of its non-destructive evaluation in the THz range (Chiou et al. 2012). Such materials seem to be ideal for the examination in the considered range of radiation, because polyethylene is highly transparent in the THz range with the low absorption coefficient varying between $0.3-1.5 \mathrm{~cm}^{-1}$ over the whole spectral range and it is almost dispersionless with the refractive index equal to 1.53 (Birch et al. 1981).

In this paper we report on the terahertz analysis of the UHMWPE composite material by means of the TDS technique in the reflection configuration. Carried out research and development studies concern the analysis of the influence of the manufacturing process parameters (temperature, pressure, etc.) on an internal structure of the composite. Therefore, there is a need for characterization of the developed materials in a non-destructive manner. The developed structures can be used to manufacture bulletproof vests and helmets.

The mechanism of the formation of many shifted in time pulses in the reflected beam is explained and it results from the modulation of the refractive index connected with the orientation of fibers in relation to the incident radiation. Next, we obtained a detailed three dimensional profile of the investigated sample with 74 clearly visible layers. The average thickness of the layer (about $45 \mu \mathrm{m}$ ) agrees well with the results obtained from an optical microscope as well as measured by a micrometer screw. Thicknesses of all layers were determined with $2-\mu \mathrm{m}$ precision. Finally, we also present the possible applications of the method to study defects in the PE multilayer structure.

Best of our knowledge, it is the first time when the internal multilayer structure of the UHMWPE composite material was determined by means of the TDS technology in such a detailed and precise manner.

\section{The characteristic of the sample}

The UHMWPE consists of extremely long chains of polyethylene with a simple repeated atomic structure (Kutz 2004). Due to intermolecular interactions, this material is very tough and have high impact strength. Spun fibers of UHMWPE can be aligned and bounded into thin strips. The photograph of the commercially available UHMWPE woven and laminated tape HB10 from Dyneema ${ }^{\circledR}$ with two such interweaving stripes in perpendicular orientation of fibers $\left(0^{\circ} / 90^{\circ}\right)$ is shown in Fig. 1a. The long HB10 roll was cut into smaller sheets which were layered and compacted to form a multilayer composite material (Figs. 1b, 2a). The configuration of the woven HB10 layers in this process is random and therefore, there are some regions with the regular (Fig. $1 \mathrm{~b}$-along the line A, $90^{\circ}-0^{\circ}-90^{\circ}-0^{\circ}$. .) and irregular (along the line $\mathrm{B}, 0^{\circ}-90^{\circ}-90^{\circ}-0^{\circ} .$. ) arrangement of layers.

The photograph of a sample having the dimensions of $50 \times 50 \mathrm{~mm}$ and a centrally located scanning area with the diameter of $30 \mathrm{~mm}$ is shown in Fig. 2a. The thickness of the sample is 

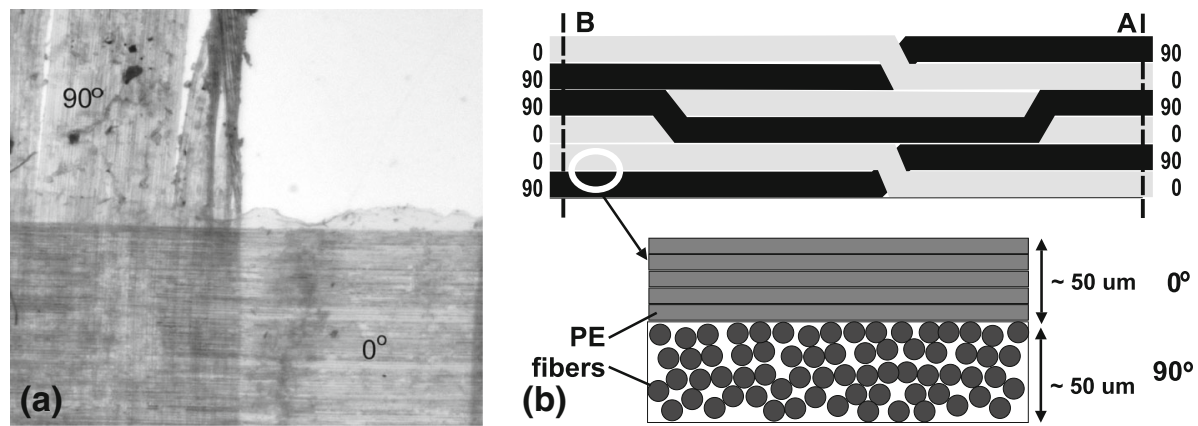

Fig. 1 The photograph of two layers of the UHMWPE composite material-front view (a) and the scheme of the HB10-based structure with magnified view of those two layers (b)
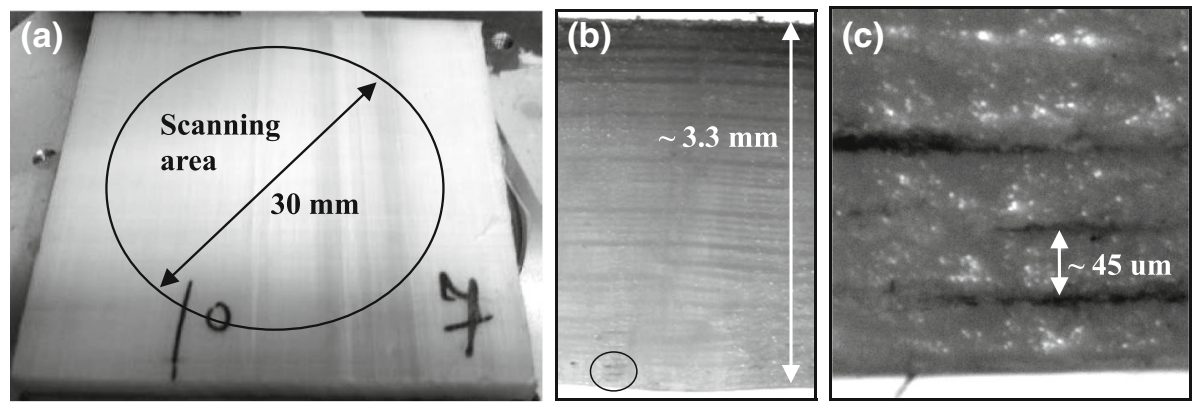

Fig. 2 The HB10-based composite material - front view (a), the lateral surface (b) and the enlargement of the lateral surface showing a few layers (c)

about $3.3 \mathrm{~mm}$ and varies along the sample in the range $\pm 0.1 \mathrm{~mm}$. The lateral surface of the composite was measured with an optical microscope (Fig. 2b). Horizontal stripes are clearly visible and they represent the layers of UHMWPE fibers. Figure $2 \mathrm{c}$ shows an enlargement of the selected area of the structure (marked with the circle in Fig. 2b). The thickness of randomly chosen single layer is marked with the arrow and it is equal to about $45 \mu \mathrm{m}$.

A piece of the manufactured material was also split into the basic layers of the HB10 tape. The thickness of a few layers was measured by means of a micrometer screw and the resulting thickness of the single layer was equal to about $40-55 \mu \mathrm{m}$. Due to the fact that the sample was separated from a larger sheet, the exact number of layers and their configuration is not known to the authors.

\section{Experimental setup}

TDS is mature and the most frequently used NDE technique in the THz range of radiation. In our investigations we used the commercially available TDS-based scanner TPS 3000 from TeraView with the XY reflection imaging module (Fig. 3a). Briefly, the scanner utilizes a $800 \mathrm{~nm}$ femtosecond Ti:Sapphire laser, two low temperature grown GaAs photoconductive antennae, a mechanical delay line and electronic controllers to emit and receive a short pulse of the electromagnetic radiation. This pulse (Fig. 3b) lasts about $0.33 \mathrm{ps}$ and has a very broadband spectrum in the range of $0.1-3.0 \mathrm{THz}$. The XY reflection imaging module enables 


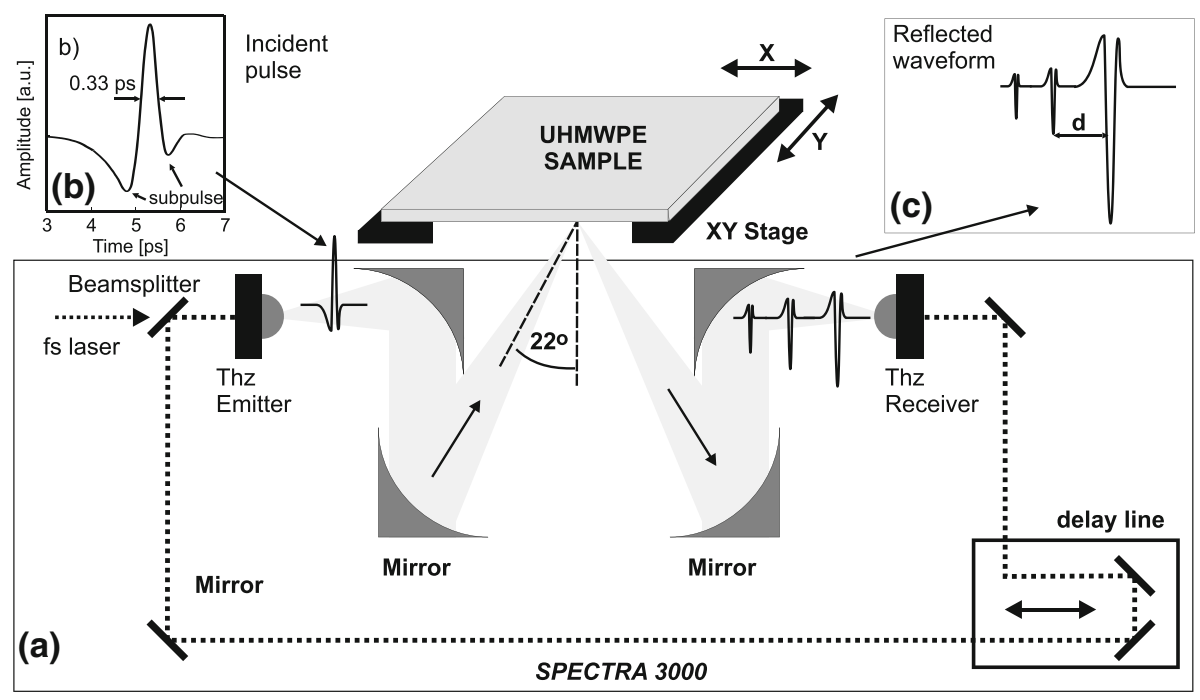

Fig. 3 TDS unit with the XY reflection imaging module: the schematic layout of the system (a), the incident pulse (b) and the reflected waveform (c)

placing samples on the top of the core unit and scanning in a raster fashion by a set of motorized stages moving in $\mathrm{X}$ and $\mathrm{Y}$ directions. The scanner is designed for rather small samples $(80 \times 80 \mathrm{~mm}$ maximum) with the pixel collection rate equal to about $60 \mathrm{msec}$. We acquired 40-ps long waveforms with 2048 points as a standard.

In the experimental setup the short THz pulse from the emitter is focused on the multilayer sample by means of a set of mirrors. The incident angle is $22^{\circ}$. The part of the pulse is reflected from the surface (Fresnel reflection), while the rest of it propagates inside the sample. If the propagating pulse encounters an interface (change of the refraction index) inside the sample, a part of it is reflected again. As a result the reflected waveform consists of the sequence of pulses reflected from the interfaces of the sample layer/layers (Fig. 3c). The pulse associated with the beginning of a dielectric sample usually has the highest amplitude in the waveform due to the fact that reflectivity is the highest at the air-sample boundary. Since the whole sample is scanned point by point, a time-of-flight analysis of the reflected waveforms can map out the internal layered structure and a three dimensional (3D) image of the sample can be obtained.

The sample is placed on a metallic holder with a hole having the diameter of $30 \mathrm{~mm}$. The central part of the sample was placed over the hole (marked with the circle in Fig. 2a) and was scanned point by point with a $250 \mu \mathrm{m}$ step. In each scanning point 10 waveforms were acquired and averaged to increase signal-to-noise ratio and to achieve higher precision. Acquisition time of the whole scan for these parameters was equal to about $30 \mathrm{~min}$. The system was purged with dry air to decrease the humidity, thus to eliminate the influence of the water vapor.

The lateral (or spatial) resolution in the TDS setups is associated with the spot size of the $\mathrm{THz}$ beam. The spot size is frequency dependent - the higher the frequency-the smaller the spot size. According to the manufacturer, the lateral resolution at the surface is equal to $160 \mu \mathrm{m}$ at $2.58 \mathrm{THz}$ and increases to about $0.3 \mathrm{~mm}$ at $1 \mathrm{THz}$. The beam propagating through the structure is being scattered. It worsens the lateral resolution, which is defined by the manufacturer as $80 \mu \mathrm{m}$ at $1-\mathrm{mm}$ depth. 
The ability to resolve two closely spaced reflections associated with adjacent layers (Fig. 3c) defines the axial resolution (d) in the TDS system. It can be determined using the following formula (Yasui et al. 2005):

$$
\mathrm{d}=(\mathrm{c} \Delta \mathrm{T}) /\left(2 \mathrm{n}_{\mathrm{g}}\right)
$$

where $\mathrm{c}$ is the velocity of light in vacuum, $\Delta \mathrm{T}$ is the temporal width of the THz pulse and $\mathrm{n}_{\mathrm{g}}$ is the group refractive index of the PE. The axial resolution in the considered optical system is estimated to be about $33 \mu \mathrm{m}\left(\Delta \mathrm{T}=0.33 \mathrm{ps}, \mathrm{n}_{\mathrm{g}}=1.53\right)$, what is consistent with the manufacturer data.

Moreover, we defined the axial precision of the system, equal to $2 \mu \mathrm{m}$, using the standard deviation of the position of the single measurement point in the reflected waveform. See inset in Fig. 7.

\section{The explanation of the propagation mechanism}

The refractive index $n(\omega)$ of the sample was determined using the TDS setup in the transmission configuration. Having the incident and transmitted $\mathrm{THz}$ amplitudes of the signal and the thickness of the sample we can easily calculate the refractive index (Chen et al. 2007). Since the index of refraction of UHMWPE is nearly constant throughout the terahertz range of interest $(0.2-3.0 \mathrm{THz})$ and is equal to 1.528 . Thus it is assumed that the group index is nominally equal to the phase index, which simplifies the analysis.

The linearly polarized $\mathrm{THz}$ wave travels through the medium with alternately arranged perpendicular and parallel layers of the thin polyethylene fibers. Such geometry of the structure causes the periodic modulation of the refractive index along the propagation of the radiation (Fig. 4b). To prove this statement, about 50- $\mu \mathrm{m}$ thick single layer of the UHMWPE fibers (all arranged in the same direction) was measured in the transmission TDS setup both for perpendicular and parallel orientation of the electric vector in relation to the direction of fibers. The refractive index for the parallel orientation $\left(\mathrm{n}_{2}\right)$ is lower than the calculated above refractive index of the multi-layer sample (n) by about 0.04 and for the perpendicular orientation $\left(\mathrm{n}_{1}\right)$ is higher by 0.04 .

If the incident wave $\left(E_{i n}\right)$ travels from a medium with the lower (higher) refractive index $\mathrm{n}_{2}\left(\mathrm{n}_{1}\right)$ into a second medium with the higher (lower) refractive index $\mathrm{n}_{1}\left(\mathrm{n}_{2}\right)$, then the signal reflected at the interface $\left(E_{r e}\right)$ has inverted (non-inverted) phase. In accordance to

(a)
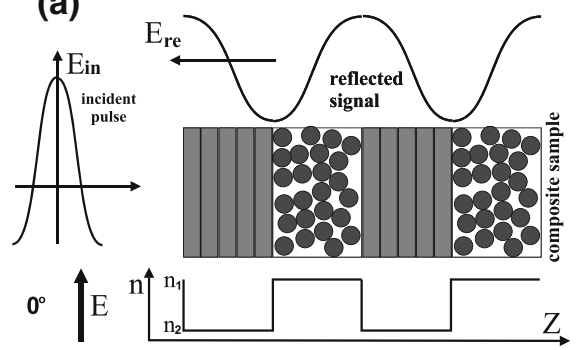

(b)

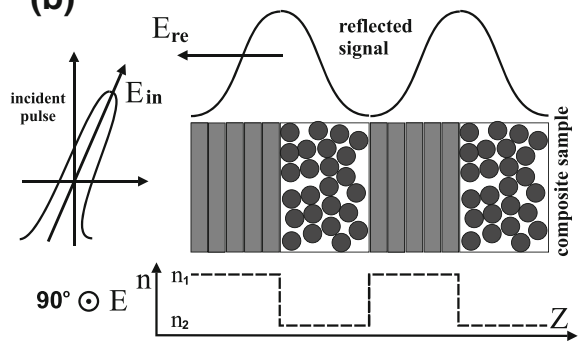

Fig. 4 Relations between the incident pulse and the reflected signal for two orientation of the E field vector in relation to the direction of the fibers in the first layer: $0^{\circ}$ (a) and $90^{\circ}$ (b) 


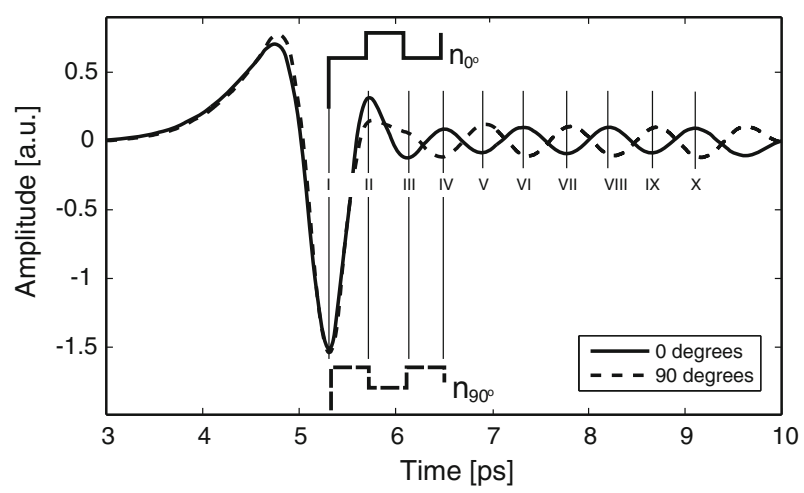

Fig. 5 The reflected pulses in two different orientations of the incident E field $-0^{\circ}$ and $90^{\circ}$ together with the diagrams showing the distribution of the refractive index in both cases $-\mathrm{n}_{0}{ }^{\circ}$ and $\mathrm{n}_{90^{\circ}}$

Fresnel equations, the regions with higher and lower refractive indices can be determined (Fig. 4).

Let us assume that the beam is incident at normal incidence on the air-sample interface and that the E field vector is parallel to the direction of the fibers in the first layer $-0^{\circ}$ (Fig. 4a). According to the above theory, if the $\mathrm{E}$ field vector is rotated by $90^{\circ}$ in relation to the normal to the surface of the sample in the point where beam incidents (Fig. 4a), the corresponding refractive index $n_{1}\left(n_{2}\right)$ changes into $n_{2}\left(n_{1}\right)$. The change of refractive indices causes the change in the way of reflection and therefore reflected waveforms for those two orientations should be in antiphase (Fig. 4).

The incident pulse and the exemplary waveforms reflected from the PE composite in relation to the orientation of the E field vector $\left(0^{\circ}\right.$ and $\left.90^{\circ}\right)$ are shown in Fig. 5. Due to the fact that the refractive index of the air is always smaller than the refractive index of the material $\left(n<n_{1}, n_{2}\right)$ we can notice that for both orientations the pulses reflected from the first interface (I) are very similar (Fig. 5).

The difference between the waveforms becomes visible for the second interface (II) (Fig. 5) The amplitude of the signal for $90^{\circ}$ orientation is significantly lower than the amplitude for $0^{\circ}$. Then for the third interface (III) the amplitude of the $0^{\circ}$ diminishes and the waveforms start to be out-of-phase.

The above described phenomenon (Fig. 4) is faintly visible for the II and III interfaces (Fig. 5) and the experimental results are inconsistent with the theory. Both pulses (having perpendicular orientations) have similar geometry of their waveforms. Problems for first three interfaces result from the fact that incident pulse (Fig. 3b) does not have ideal shape (e.g. Gaussian distribution) but contains also some subpulses. At the beginning their amplitudes are comparable with the amplitude of the signal reflected from the second interface (II). Due to this fact the reflected pulse is concealed in the subpulses from the incident signal. The same situation partially takes place for the third interface (III).

Not before the reflection from the fourth interface (IV) the theory corresponds to the experimental results. In this case the influence of the subpulses can be neglected, because they become very small in comparison to the reflected signal. In accordance with the described theory the two waveforms (having perpendicular orientations) are in antiphase (it takes place for the time longer than $6 \mathrm{ps).} \mathrm{Small} \mathrm{differences} \mathrm{probably} \mathrm{result} \mathrm{from} \mathrm{the} \mathrm{fluctuations} \mathrm{of}$ the refractive index caused by the technological scatter in the manufacturing process of the composite. 
(a)

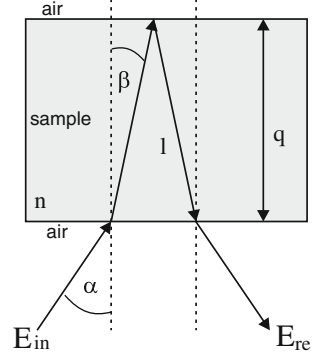

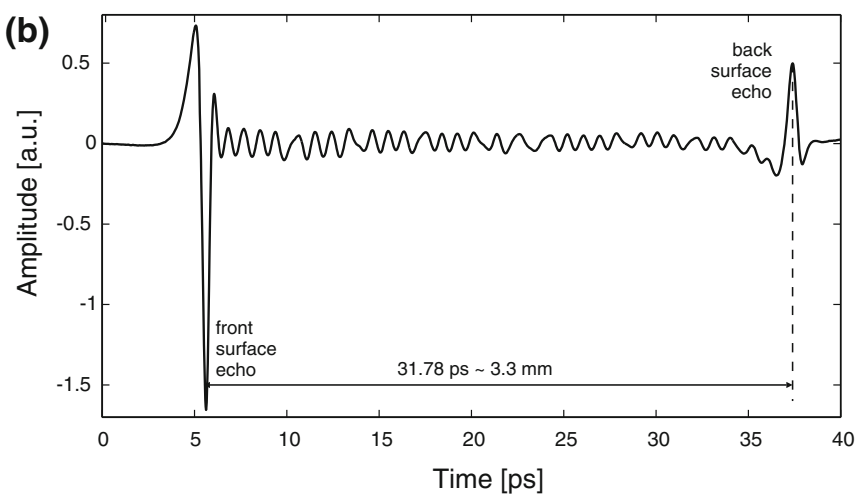

Fig. 6 Diagram showing the geometry of the reflection and the refraction (a) and the waveform of the pulse reflected from the sample (b)

\section{Determination of the thickness of each layer}

The diagram with the geometry of the reflection at the surface of the sample and the refraction inside it (for the considered optical setup) is presented in Fig. 6a. The incident angle $(\alpha)$ and the refraction index $(n)$ of the sample are known $\left(22^{\circ}\right.$ and 1.528 , respectively), thus by means of the Snell's law the refraction angle $(\beta)$ can be easily calculated. In our experiment $\beta$ is equal to $14^{\circ}$. Since the angle of refraction is not equal to 0 , the sample thickness $q=3.3 \mathrm{~mm}$ must by multiplied by 1.03 to determine a real one-way path of the THz pulse through the sample $(l)$, which is equal to $3.4 \mathrm{~mm}$.

The waveform reflected from the examined sample is shown in Fig. 6b. It can be noticed that the first high and inverted pulse is the reflection from the front layer of the sample and the second high pulse is associated with reflection from the back layer of the sample. Between these pulses there are many oscillations that are related to the internal structure of the sample.

A detailed plot of the oscillation region is shown in Fig. 7. The time difference on the reflected pulse between the reflection from the front and the back layers of the sample is equal to $31.78 \mathrm{ps}$ and the thickness of the sample is equal to $3.3 \mathrm{~mm}$. Taking into account the factor 1.03, one can easily find that $1 \mathrm{ps}$ corresponds to about $104 \mu \mathrm{m}$ and thicknesses of all 63 detected regions with alternately changing refractive index can be calculated. These regions correspond to subsequent layers of fibers with two different orientations (parallel and perpendicular to the incident E field vector). Inset in Fig. 7 shows a short segment of the

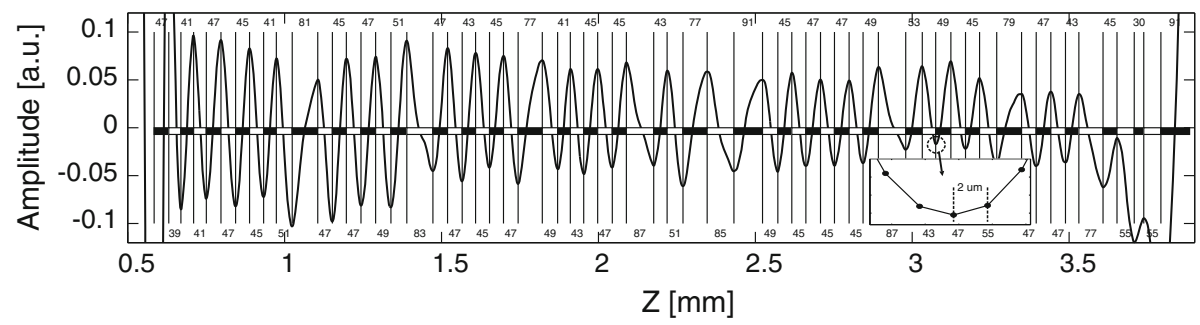

Fig. 7 The reflected pulse with the thickness of each layer marked in $\mu \mathrm{m}$. Dark and bright rectangles represent the regions with lower and higher refraction indices, respectively. Thicknesses of all layers are presented above and below the waveform. Inset shows the zoom of the waveform with the measuring points $(2-\mu \mathrm{m}$ precision) 

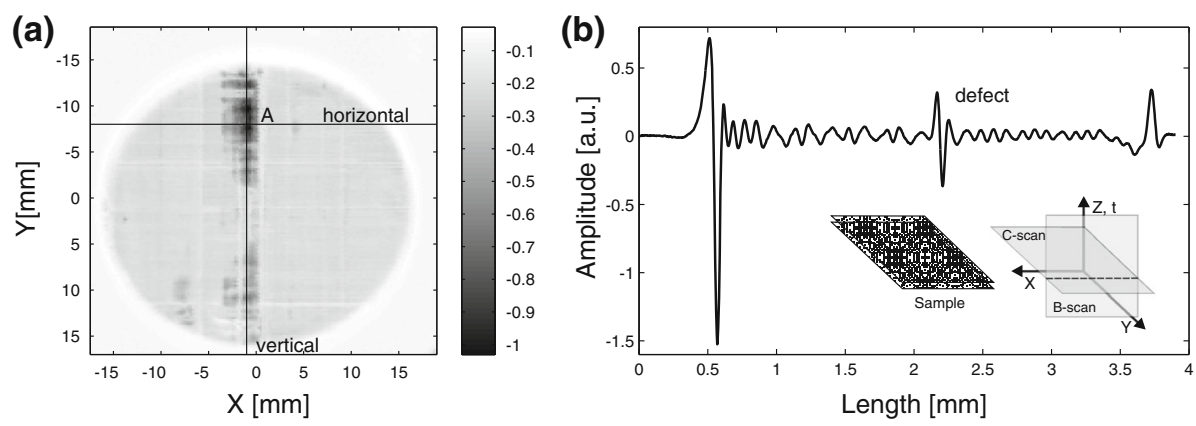

Fig. 8 The exemplary C-scan with the peak-to-peak analysis (a) and the waveform at the A point with the defect-related pulse (b). Inset shows the idea of B- and C-scans

waveform with measuring points. The distance between the consecutive points is equal to $2 \mu \mathrm{m}$.

It can be noticed from Fig. 7 that thickness of the single layer is usually between 40 and $50 \mu \mathrm{m}$, which corresponds well to the results presented above. We consider, that values in the range 77-91 $\mu \mathrm{m}$ are associated with two adjacent layers of fibers arranged in the same direction (Fig. 1b-line B). Near the back surface of the sample we can see that the thickness of only one layer is equal to $30 \mu \mathrm{m}$ and it probably results from the minor uniformity of the manufacturing process. Assuming that 11 layers (having the thickness above $77 \mu \mathrm{m}$ ) consist of two single layers, the total number of layers is equal to 74 .

Additionally, it can be concluded that the process of the composite material manufacturing is uniform and therefore the layers in the middle of the sample have similar thickness as those near the surfaces of the sample.

\section{Analysis of the defects}

A 3D image with $\mathrm{X}$ and $\mathrm{Y}$ axes representing the position and $\mathrm{Z}$ axis representing the optical delay time can be obtained as a result of the scanning. To analyze such images in two dimensional space the two sets of cross-section views were introduced: the B-scan, perpendicular to the surface of the sample (X-t or Y-t cut) and the C-scan, parallel to the surface of the sample (X-Y cut) - see inset in Fig. 8b.

The C-scan (Fig. 8a) with a peak-to-peak (Pk-Pk) analysis in the depth interval ( $\mathrm{Z}$ axis) from 0.7 up to $3.5 \mathrm{~mm}$ was carried out to reveal regions with higher signal. $\mathrm{Pk}-\mathrm{Pk}$ means the ratio between the maximum and the minimum values in an arbitrary defined time interval. Darker regions in the central part of the Fig. 8a indicate regions with defects. The waveform at the A point corresponds to the high pulse associated with a defect in the middle of the structure (Fig. 8b) - probably a small delamination between the layers. Reflections at the interfaces composite/air and air/composite are higher in comparison to reflections at the internal interfaces of the composite material.

The horizontal and vertical B-scans along the lines marked in Fig. 8a are depicted in Fig. 9a, b, respectively. The peaks reflected from the surface (at about $0.5-0.6 \mathrm{~mm}$ ) were artificially suppressed to enhance interesting features (corresponding to defects) inside the sample. The position and the size of the defect at about $2.3-2.6 \mathrm{~mm}$ is visible for both Bscans. By choosing other lines or points on the surface of C-scan (see Fig. 8a with the marked 

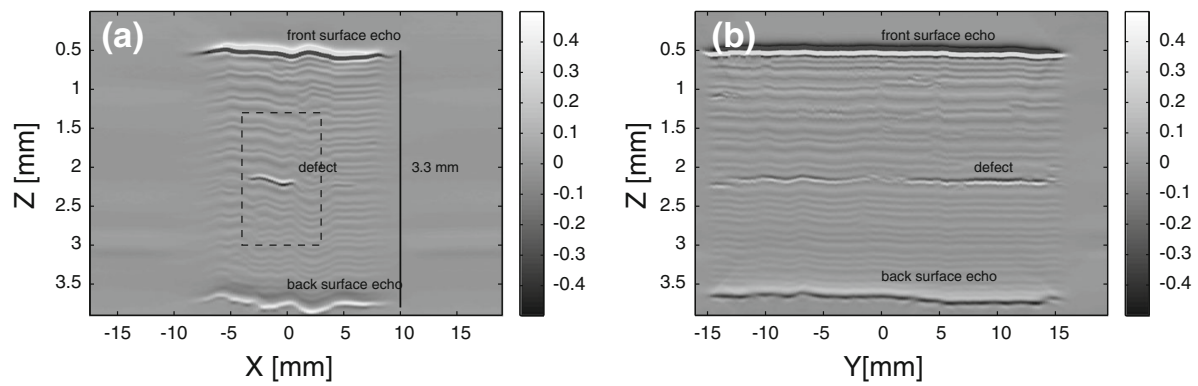

Fig. 9 The B-scan: along the horizontal (a) and the vertical (b) lines with the defect visible in the middle of the sample

A point and two lines along which the B-scans were carried out) it is possible to analyze the defect more precisely.

One can notice that the layers are rather flat in case of the B-scan along the vertical line (Fig. 9b). A more complicated pattern visible for the B-scan along the horizontal line (Fig. 9a) at about $\mathrm{X}=1 \mathrm{~mm}$ probably results from the fact that in this position the fibers of the HB10 tape change their orientation. The two B-scans together with the $\mathrm{C}$-scan allows us to remark that the defect is the delamination.

\section{Summary}

We reported on the non-destructive evaluation of the UHMWPE composite material (HB10tape from Dyneema $\left.{ }^{\circledR}\right)$. The method uses nonionizing radiation and allows for the contactless examination even of nonconductive materials. It also gives the possibility of determining the internal structure of the composite to exclude any defects which is crucial due to its application in manufacturing personal armors. The possibilities offered by THz TDS technique in the reflection configuration to examine the internal structure and to detect various defects were demonstrated by scanning the 3.3-mm thick sample of the UHMWPE. The mechanism of the formation of reflections inside the sample from subsequent fiber layers with alternate orientation (one perpendicular to the next one) was described. Due to the periodic modulation of the refractive index along the propagation of the radiation connected with the change of the direction of fibers in the layers, the described method enabled determining the quantity and the thicknesses of the internal composite layers. The carried out measurements enabled us to determine thicknesses of all 74 layers, each having around $45 \mu \mathrm{m}$, with the precision of 2$\mu \mathrm{m}$. Moreover, we showed that it is possible to study the internal structure of this composite material and identify any heterogeneities of the sample with no negative influence on it. Internal defects i.e. delaminations can be easily discovered without destroying the sample, with no harm to operators (as opposed to X-rays) and with the minimal possibility of the human error (in contrast to video examination). The position of each defect can be defined very precisely and some additional scans can be carried out to help to qualify the type of the defect when more information is needed.

THz Time Domain Spectroscopy technique allowed us to precisely examine the internal structure of the UHMWPE with defining the subsequent layers of fibers and their thicknesses. Moreover, different imperfections occurring in the internal structure can be found, which is crucial for safety in using different objects manufactured from UHMWPE (like personal armors). 
Acknowledgments The paper supported by Grant No NR O ROB 0012 02, financed in the years 2012 2014 by The National Centre for Research and Development, Poland. We acknowledge helpful discussion with Roman Gieleta.

Open Access This article is distributed under the terms of the Creative Commons Attribution License which permits any use, distribution, and reproduction in any medium, provided the original author(s) and the source are credited.

\section{References}

Bathias, C., Cagnasso, A.: Application of X-ray tomography to the nondestructive testing of high-performance polymer composites. In: Masters, J.E. (ed.) Damage Detection in Composite Materials, pp. 35-54. American Society for Testing and Materials, Fredericksburg (1992)

Birch, J.R., Dromey, J.D., Lesurf, J.: Optical constants of some low-loss polymers between 4 and $40 \mathrm{~cm}-1$. Infrared Phys. 21, 225-228 (1981)

Chan, W., Deibel, J., Mittleman, D.: Imaging with terahertz radiation. Rep. Prog. Phys. 70, 1325-1379 (2007)

Chen, J., Chen, Y., Zhao, H., Bastiaans, G.J., Zhang, X.-C.: Absorption coefficients of selected explosives and related compounds in the range of 0.1-2.8 THz. Opt. Express 15, 12060 (2007)

Chiou, C.-P., Margetan, F.J., Barnard, D.J., Hsu, D.K., Jensen, T., Eisenmann, D.: Nondestructive characterization of UHMWPE armour materials. AIP Conf. Proc. 1430, 1168-1175 (2012)

Destic, F., Petitjean, Y., Massenot, S., Mollier, J.-C., Barbieri, S.: THz QCL-based active imaging dedicated to non-destructive testing of composite materials used in aeronautics. Proc. SPIE 7763, 776304 (2010)

Dragan, K., Swiderski, W.: Studying effciency of NDE techniques applied to composite materials in aerospace applications. Acta Phys. Pol. A. 117, 878-883 (2010)

Gendron, R., Tatibouët, J., Guèvremont, J., Dumoulin, M.M., Piché, L.: Ultrasonic behavior of polymer blends. Polym. Eng. Sci. 35, 79-91 (1995)

Hughes, D., Kazemi, M., Marler, K., Zoughi, R., Myers, J., Nanni, A.: Microwave detection of delaminations between fiber reinforced polymer (FRP) composite and hardened cement paste. AIP Conf. Proc. 615(1), 512-519 (2002)

Karpowicz, N., Zhong, H., Zhang, C., Lin, K., Hwang, J.S., Xu, J., Zhang, X.-C.: Compact continuous-wave sub-terahertz system for inspection applications. Appl. Phys. Lett. 86, 054105 (2005)

Kutz, S.M.: The UHMWPE Handbook: Ultra-High Molecular Weight Polyethylene in Total Joint Replacement. Elsevier, San Diego (2004)

Levar, J.M., Hamilton, H.R.: Nondestructive evaluation of carbon fiber-reinforced polymer-concrete bond using infrared thermography. ACI Mat. J. 100-M8, 63-72 (2003)

Lopato, P., Chady, T., Goracy, K.: Image and signal processing algorithms for THz imaging of composite materials. AIP Conf. Proc. 1211, 766-773 (2009)

Mittleman, D.M., Gupta, M., Neelamani, R., Baraniuk, R.G., Rudd, R.G., Koch, M.: Recent advances in terahertz imaging. Appl. Phys. B 68, 1085-1094 (1999)

Quast, H., Kiel, A., Hoyer, T., Loeffler, T.: All-electronic 3D terahertz imaging for the NDT of composites. In: 2nd International Symposium on NDT in Aerospace 2010-We.4.B.3 (2010)

Redo-Sanchez, A., Karpowicz, N., Xu, J.: Damage and defect inspection with terahertz waves. In: The 4th International Workshop on Ultrasonic and Advanced Methods for Nondestructive Testing and Material Characterization, pp. 67-78 (2006)

Rutz, F., Wietzke, Koch, M., Richter, H., Hickmann, S., Trappe, V., Ewert, U.: Non-destructive testing of glassfibre reinforced polymers using terahertz spectroscopy. In: 9th European Conference on Non-Destructive Testing, Sept. 2006, Berlin, Germany (2006)

Stoik, C., Bohn, M., Blackshire, J.: Nondestructive evaluation of aircraft composites using reflective terahertz time domains spectroscopy. NDT\&E Int. 43, 106-115 (2010)

Takayanagi, J., Jinno, H., Ichino, S., Suizu, K., Yamashita, M., Ouchi, T., Kasai, S., Ohtake, H., Uchida, H., Nishizawa, N., Kawase, K.: High-resolution time-of-flight terahertz tomography using a femtosecond fiber laser. Opt. Express 17, 7533 (2009)

Wietzke, S., Jordens, C., Krumbholz, N., Baudrit, B., Bastian, M., Koch, M.: Terahertz imaging: a new nondestructive technique for the quality control of plastic weld joints. J. Eur. Opt. Soc. 2, 07013 (2007)

Yasui, T., Yasuda, T., Sawanaka, K., Araki, T.: Terahertz paintmeter for noncontact monitoring of thickness and drying progress in paint film. Appl. Opt. 44, 6849 (2005) 